\title{
Twisted bilayer graphene aligned with hexagonal boron nitride: Anomalous Hall effect and a lattice model
}

\author{
Ya-Hui Zhang ๑, Dan Mao, and T. Senthil \\ Department of Physics, Massachusetts Institute of Technology, Cambridge, Massachusetts 02142, USA
}

(Received 12 February 2019; revised manuscript received 24 October 2019; published 25 November 2019)

\begin{abstract}
A recent experiment reported a large anomalous Hall effect in magic angle twisted bilayer graphene (TBG) aligned with a hexagonal boron nitride (h-BN) substrate at $3 / 4$ filling of the conduction band. In this paper we study this system theoretically and propose explanations of this observation. We emphasize that the physics for this system is qualitatively different from the pure TBG system. The aligned h-BN breaks in-plane twofold rotation symmetry and gaps out the Dirac crossings of ordinary TBG. The resulting valence and conduction bands of each valley carry equal and opposite Chern numbers $C= \pm 1$. A useful framework is provided by a lattice extended Hubbard model for this system, which we derive. An obvious possible explanation of the anomalous Hall effect is that at $3 / 4$ filling the system is a spin-valley polarized ferromagnetic insulator where the electrons completely fill a Chern band. We also examine an alternate, more radical proposal of a compressible valleypolarized but spin-unpolarized composite Fermi-liquid metallic state. We argue that either state is compatible with current experiments and propose ways to distinguish between them in the future. We also briefly discuss the physics at $1 / 2$ filling.
\end{abstract}

DOI: 10.1103/PhysRevResearch.1.033126

\section{INTRODUCTION}

Moiré superlattices from twisted van der Waals heterostructures have emerged as promising platforms to study strongly correlated effects with high tunability [1-5]. Correlated insulators and superconductors have been found in twisted bilayer graphene and $\mathrm{ABC}$ stacked trilayer graphene/hexagonal boron nitride (TG/h-BN) [2-5].

Very recently a large anomalous Hall effect was observed [6] in magic angle-twisted bilayer graphene (MA TBG) at conduction-band filling $v=\frac{3}{4}$. Specifically, hysteretic jumps in both the Hall resistivity $\left(\rho_{x y}\right)$ and longitudinal resistivities $\left(\rho_{x x}\right)$ were observed. At the lowest temperatures, the maximal measured Hall resistivity is $\rho_{x y} \approx 0.5 \frac{h}{e^{2}}$ and the corresponding resistivity is $\rho_{x x} \approx 0.3 \frac{h}{e^{2}}$, corresponding to a large Hall angle. Evidence for nonlocal transport, indicative of conducting channels at the sample edge, have been presented. A key new feature of the device studied in Ref. [6] is that one of the graphene layers is nearly aligned with a h-BN substrate. This alignment has many important effects, as we explain below, and serves to distinguish this system from previous experiments on magic angle TBG where no such anomalous Hall effect has been reported.

In this paper we study theoretically the MA TBG-hBN system and propose possible explanations of these observations. A spontaneously spin-valley polarized Chern insulator

Published by the American Physical Society under the terms of the Creative Commons Attribution 4.0 International license. Further distribution of this work must maintain attribution to the author(s) and the published article's title, journal citation, and DOI. at 3/4 filling provides a simple and natural explanation for the large anomalous Hall effect. We also consider a different novel state which may also explain the data-a compressible composite Fermi-liquid metal with valley polarization but no spin polarization. We propose experiments to distinguish these two distinct states.

In the absence of alignment with h-BN, the moiré band structure of MA-TBG has "active," nearly flat bands that are well separated from other bands. The active bands live in each of two mini Brillouin zones (MBZs) corresponding to the two valleys of the underlying graphene layers. Within each valley the conduction and valence active bands are connected by Dirac points at the corners of the MBZ. These Dirac points are protected by an excellent emergent $C_{2} T$ symmetry [7], where $C_{2}$ refers to a twofold rotation and $T$ is time reversal. Either $C_{2}$ or $T$ maps one valley to the other, but their combination preserves the valley index. This $C_{2} T$ symmetry maps nonzero Berry curvature $B(\mathbf{k})$ to $-B(\mathbf{k})$ and thus forbids nonzero mass terms for the Dirac crossings. If, however, $C_{2} T$ is broken then the Dirac points will become gapped. Experimentally, the presence of Dirac points is evidenced by studying the properties of the system filled to the charge neutrality point (CNP). Typically at CNP the system is metallic with a low but nonzero conductance.

An important effect of alignment with h-BN is that the broken $C_{2}$ symmetry of h-BN is transmitted to the graphene bands. Thus the Dirac points are gapped and insulating behavior may be obtained at the CNP. This is supported by the measured $\rho_{x x}$ at neutrality in Ref. [6], which is much bigger than the typical measured values in unaligned TBG devices. Furthermore, the resulting isolated conduction and valence bands in each valley carry Chern numbers $C=1,-1$ (opposite valleys carry opposite Chern numbers). Thus the 
MA TBG-hBN is similar to the many other examples of nearly flat $\pm C$ bands discussed theoretically recently [8]. As emphasized in Ref. [8], at total fillings $\nu_{T}=1,3$ nearly flat \pm Chern bands are an excellent platform for the quantum anomalous Hall effect, as well as other even more novel many-body states. Recently Ref. [9] described a spin-valley polarized quantum anomalous Hall state in unaligned twisted bilayer graphene where $C_{2} T$ is broken by interaction effects.

A further effect of the alignment with h-BN is that there are now two distinct moiré superlattices. In addition to the moiré potential produced by the relative twisting of the two graphene layers, the lattice mismatch between h-BN and graphene produces another moiré potential [10]. These two moiré lattices have roughly the same period but are rotated by $90^{\circ}$ relative to each other, which makes them mutually incommensurate. However, the strength of the h-BN-induced moiré potential is expected to be weaker than the other one, and it is a reasonable approximation to ignore it to begin with. It may, however, play a role by producing in-gap states that may contribute to the lack of exact quantization of the Hall resistivity (in addition to other mechanisms involving disorder) in the experiments.

The experimental developments on correlated moiré superlattices has spawned a large theoretical literature-for a sample see Refs. [11-55]. An important conclusion $[12,36,37,48,49]$ is that the bands of TBG have a (symmetry) protected topological structure which obstructs the construction of lattice tight-binding models with natural ("on-site") action of all symmetries. The $C_{2} T$ breaking induced by the alignment with h-BN, however, removes this obstruction, and it is possible to construct a lattice tight-binding model to represent the conduction and valence band taken together within each valley. Unsurprisingly, we show that this takes the form of a lattice Haldane model. Combining the two valleys and projecting the Coulomb interaction yields an effective lattice "extended" Hubbard model suitable for TBG-hBN. This lattice model provides a useful framework to discuss the physics and may also be useful for future numerical studies.

\section{BAND STRUCTURE}

We consider a twisted bilayer graphene where the top layer is aligned with the h-BN layer substrate. The twist angle between the two graphene layers $\theta_{M}$ is chosen to be close to the magic angle $\theta_{M}=1.05^{\circ}-1.20^{\circ}$. The twist angle between the top h-BN layer and the top graphene layer $\theta_{h B N}$ is close to zero. We assume the bottom h-BN substrate is misaligned and its effect can be ignored.

We use the standard continuum model [56] (with $\frac{w_{0}}{w_{1}}=0.7$ [13] to account for lattice relaxation) to calculate the band structure of the TBG/h-BN system. As time-reversal symmetry flips the valley, we can focus only on the band structure within a single valley, say + . The Hamiltonian is

$$
H=H_{T B G}+H_{h B N} .
$$

Here $H_{T B G}$ is the continuum model for the TBG in Ref. [56]. The aligned h-BN has two effects on the top graphene

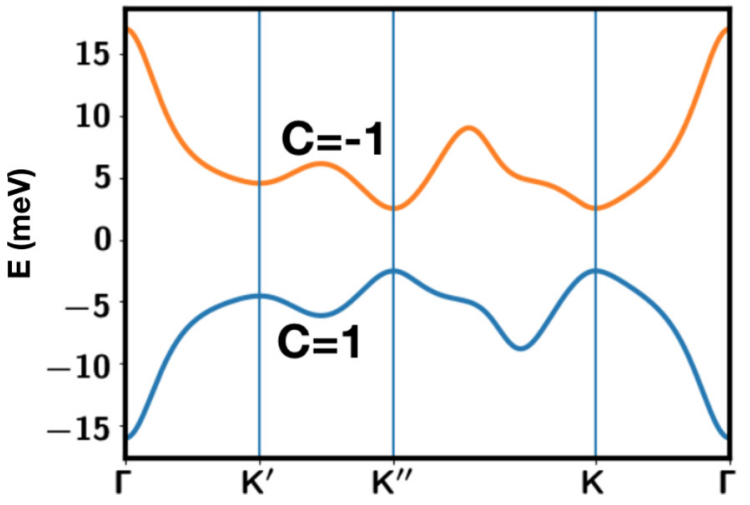

FIG. 1. Band structure for valley + of the TBG/h-BN system in the MBZ. $\theta_{M}=1.20^{\circ}$. The band of valley - can be generated from the time-reversal transformation.

layer:

$H_{h B N}=\sum_{\mathbf{k}} M \psi_{t}^{\dagger}(\mathbf{k}) \mu_{z} \psi_{t}(\mathbf{k})+\sum_{j=1, \ldots, 6} \psi_{t}^{\dagger}\left(\mathbf{k}+\mathbf{Q}_{\mathbf{j}}^{\prime}\right) V_{j} \psi_{t}(\mathbf{k})$,

where $\psi_{t, b}(\mathbf{k})$ represents electron destruction operators in the top and bottom valley. $\mathbf{k}$ belongs to the whole $R^{2}$ space. The first term is an induced staggered potential on the $A, B$ sublattices of the top graphene layer which acts as a "mass" term. A rough estimate is obtained from experiments on monolayer graphene nearly aligned with h-BN [57] which show that the band gap at the neutrality point is around $35 \mathrm{meV}$. This implies $M \approx 17 \mathrm{meV}$. The second term in Eq. (2) represents the moiré potential coming from the lattice mismatch between h-BN and graphene. The resulting moiré wave vectors are incommensurate with those associated with the TBG superlattice. Furthermore, a rough estimate from DFT calculations gives $V_{j} \approx 10 \mathrm{meV}$ [10], which is much smaller than the strength of the TBG moiré term (around $110 \mathrm{meV}$ [56]), and somewhat smaller than the first term. Thus as a first approximation we ignore the $V_{j}$. This considerably simplifies our analysis, as we now have a well-defined band structure in the moiré superlattice of TBG.

The band structure is shown in Fig. 1 for $M=15 \mathrm{meV}$. As expected, there is a finite band gap at around $5 \mathrm{meV}$ for the value of $M$ we used. Importantly, through explicit calculation the conduction and the valence bands for the valley + has Chern number $C=1,-1$. This Chern number is a simple symptom of the underlying subtle band topology $[12,36,37,48,49]$ of the unaligned TBG system and is closely related to the "flipped Haldane model" picture described in Ref. [36].

Under a very small out of plane magnetic field, the response of a massive Dirac cone at point $K$ or $K^{\prime}$ has two contributions: (1) the first part is just the nonrelativistic response, and (2) the second part is a valley Zeeman coupling $-g_{v}(\mathbf{k}) \mu_{B} H_{z} \frac{\tau_{z}}{2}$ [58], where $\tau_{a}, a=x, y, z$ is the Pauli matrix in valley space. We numerically calculated $g_{v}$ for $\theta_{M}=1.20^{\circ}$ and $M=15 \mathrm{meV}$. Indeed, we find that the average valley Zeeman coupling $g$ factor is $\bar{g}_{v}=\frac{1}{A_{B Z}} \sum_{\mathbf{k}} g_{v}(\mathbf{k}) \approx 4$. This implies that the two valleys have an opposite out-of-plane 
magnetic moment, which is crucial to understanding the hysteresis of the anomalous Hall effect in Ref. [6]. The splitting of bands between the two valleys is also crucial in understanding the Landau fan degeneracy and the magnetoresistance. However, we need to emphasize that both contributions need to be included. Although for an infinitesimal field it is sufficient to consider the valley Zeeman coupling, in reality, the most efficient way to understand the response to $B_{z}$ is to calculate Landau levels directly. We will take this approach later to understand the magnetoresistance at $v=\frac{1}{2}$.

Combining the two valleys, we can now project the Coulomb interaction onto these bands to obtain an effective model. There are three important energy scales: the bandwidth $W$ of the conduction band, the band gap $\Delta$ (between conduction and valence bands), and the interaction strength $U$. Our focus is on the experimentally observed correlated insulators at $v=1 / 2$ and $v=3 / 4$ of the conduction band.

First consider the limit $\Delta \gg U \gg W$. Then we only need to keep the four conduction bands (including spin and valley) and the problem reduces to the nearly flat \pm Chern band system studied in Ref. [8]. In the flat-band limit, the ground state should be a ferromagnetic insulator from spin or valley polarization. In particular, valley polarization is favored over intervalley coherence within a Hartree-Fock calculation [8]. For $v=\frac{3}{4}$, the quantum anomalous Hall effect(QAHE) with $\left|\sigma_{x y}\right|=\frac{e^{2}}{h}$ emerges by polarizing both spin and valley. At $v=\frac{1}{2}$ in this flat-band limit, we expect instead a spinpolarized insulator with a quantum valley Hall effect.

\section{LATTICE MODEL}

Strictly speaking, the TBG/h-BN system is in a different limit $U \sim W>\Delta$, and the detailed many-body physics may differ from that discussed in Ref. [8]. When $\Delta<W$, both the conduction bands and the valence bands should be kept in the low-energy model. Below we provide a lattice model by Wannier construction of the active bands (conduction and valence bands) which, in contrast to standard TBG, is possible given the broken $C_{2} T$ symmetry. The system still has a $C_{3}$ rotation symmetry, and we numerically calculate the $C_{3}$ eigenvalues at the high-symmetry momentum points from the continuum model. We take the rotation center as the AA site. The $C_{3}$ eigenvalues at $\Gamma, K, K^{\prime}$ are $1, \omega, \omega$ for the conduction band and $1, \omega^{*}, \omega^{*}$ for the valence band, with $\omega=e^{i \frac{2 \pi}{3}}$. The distinct eigenvalues at $\Gamma, K, K^{\prime}$ imply that we cannot represent the system on the natural triangular lattice formed by the AA regions. A honeycomb representation is, however, possible (see Fig. 2). The corresponding Wannier functions are readily constructed and have the familiar fidget-spinner shape [12], reflecting the concentration of charge in the AA regions. We obtained the Wannier orbitals and tight-binding parameters using the standard numerical method, similar to the method in Ref. [59].

Let $\mathbf{a}_{1}=a_{M}(0,1)$ and $\mathbf{a}_{2}=a_{M}\left(\frac{\sqrt{3}}{2}, \frac{1}{2}\right)$ be two basis vectors for the honeycomb lattice and define the electron operator $c_{i ; a \sigma}$, where $a= \pm$ and $\sigma=\uparrow, \downarrow$ are the valley and spin index.

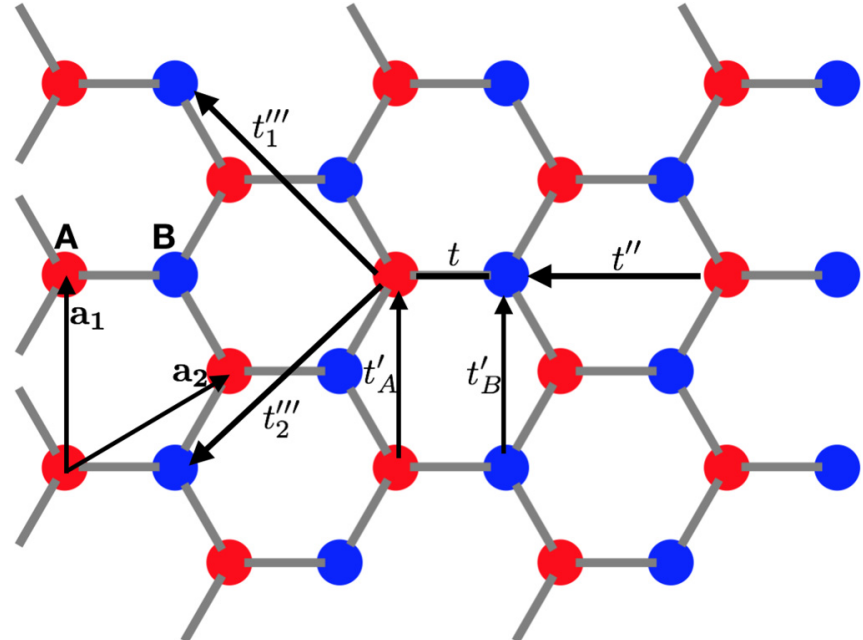

FIG. 2. Illustration of the lattice model on a Honeycomb lattice. $C_{3} t(\mathbf{R}) C_{3}^{-1}=t\left(C_{3} \mathbf{R}\right)$ generates intersublattice hopping, and $C_{6} t^{\prime}(\mathbf{R}) C^{-1}(6)=t^{\prime *}\left(C_{6} \mathbf{R}\right)$ generates intrasublattice hoppings.

The tight-binding model takes the form

$$
\begin{aligned}
H_{K}= & -m_{0} \sum_{i}(-1)^{X(i)} c_{i ; a \sigma}^{\dagger} c_{i ; a \sigma} \\
& -\sum_{a \sigma} \sum_{i j}\left(t_{i j}^{a} c_{i ; a \sigma}^{\dagger} c_{j ; a \sigma}+\text { H.c. }\right),
\end{aligned}
$$

where $X(i)= \pm 1$ on the $A$ and $B$ sublattices. Time-reversal symmetry requires that $t_{i j}^{+}=t_{i j}^{-*}=t_{i j}$. For each valley this is a modified Haldane model in its topological phase (with a few extra hopping parameters). The tight-binding parameters can be found in Appendix B.

The fidget-spinner Wannier orbital implies that the interaction is dominated by a cluster charging [12] Hubbard interaction. Furthermore, due to the nonzero spatial overlap between Wannier orbitals on different sites, there will be an intersite Hund's term $J[59,60]$. The interaction term is thus

$$
H_{V}=U \sum_{\square} n_{\square}^{2}-J \sum_{i j} \sum_{p} S_{i}^{p} S_{j}^{p}+\cdots .
$$

Here $n_{\square}$ is the electron charge summed over the sites of a hexagonal cluster [61]. $p=1, \ldots, 15$ is summed over the 15 generators of SU(4), and the ellipses represent other terms (e.g., a pair hopping) that are less important in the insulator.

Finally, we have a lattice model by combining the kinetic and the interaction terms [Eqs. (3) and (4)]. Strictly speaking, we also need to add a quasiperiodic potential from the incommensurate h-BN layer (see details in Appendix).

Given this lattice model, we can consider the strongcoupling limit $U \gg \Delta, W$. Then at integer total fillings we will get Mott insulators where the charge on every cluster is frozen. The corresponding insulator cannot have any Hall response: for a nonzero $\sigma_{x y}$, a Laughlin type of threaded flux induces charge $Q=\sigma_{x y} e$, while in the $U \gg \Delta, W$ limit the local density cannot be changed. Of course, the experimental 
system is likely in the regime $U \sim W \gg \Delta$ and this strongcoupling limit is not directly relevant.

\section{ANOMALOUS HALL EFFECT AT $v=\frac{3}{4}$}

The observation of a hysteretic anomalous Hall effect in the experiment [6] clearly shows [62] that there is spontaneous time-reversal breaking associated with valley polarization at $v=\frac{3}{4}$. Assuming full valley polarization, we then have a spinful "Haldane model" supplemented with interactions at half filling of the conduction band.

If the ground state is fully spin and valley polarized, then we get the Chern insulator and there will be a quantized anomalous Hall effect. In an ideal sample, this state has $\rho_{x y}= \pm \frac{h}{e^{2}}, \rho_{x x}=0$, corresponding to a Hall angle of $90^{\circ}$. Such a spin-valley ferromagnetic insulator state has very good Coulomb energy but has poor kinetic energy. Thus when $W \sim U$ it is interesting to contemplate other states of matter. We will assume full valley polarization in the discussion below.

For $W \gg U$, a simple Fermi liquid will be the ground state. This state has $\sigma_{x x} \gg e^{2} / h, \sigma_{x y} \sim e^{2} / h$. The Hall conductance is due to a quasiparticle Berry phase that will exist at generic filling of the Chern band. It follows that $\rho_{x y} \ll \rho_{x x}$ so that the anomalous Hall resistivity is small, unlike in the experiments.

How should we connect the Fermi liquid at $W \gg U$ to the ferromagnetic Chern insulator? A natural possibility (accessed through a Stoner mean-field theory) is that the Fermi liquid first undergoes a transition to a partially spin-polarized Fermi liquid, which then gives way at larger interaction strengths to the fully spin-polarized ferromagnetic insulator. The properties of the partially spin-polarized metal will interpolate continuously between those of the spin-unpolarized Fermi liquid and the ferromagnetic insulator. A key experimental signature of this phase will be the presence of two distinct oscillation frequencies (corresponding to the spin-split Fermi surfaces) in Shubnikov-de Haas ( $\mathrm{SdH})$ experiments.

We now describe an alternate possibility for an intermediate coupling phase. We reason by analogy to a Landau level to which a $C=1$ band is closely analogous. Since each spin species is at half filling of the $C=1$ band, we may expect the system to be similar to that of spinful electrons in a half-filled Landau level. In the traditional half-filled Landau level it is well known that a compressible metallic state-the composite Fermi liquid (CFL) - is formed. In the presence of spin it is favorable to instead spin polarize to form a ferromagnetic integer quantum Hall state. In contrast to the traditional Landau level, the Chern band has a dispersion. Thus a spin-unpolarized CFL may be competitive. Such a state should retain much of the kinetic energy of the simple Fermi liquid while doing better on the Coulomb energy. A convenient description is through a parton construction $c_{i ; \sigma}=$ $b f_{i ; \sigma}$ where the spinless slave boson $b$ carries physical charge and $f_{\sigma}$ is spin- $1 / 2$ neutral fermion. In the CFL phase, the boson is at filling $v=1$ of a $C=1$ band and can form a fractional Chern insulator (Pfaffian state) with $\sigma_{x y}^{b}=\frac{e^{2}}{h}, \sigma_{x x}=$ 0 . Using the Ioffe-Larkin rule [63], we get the resistivity tensor of the physical electrons: $\rho^{c}=\rho^{f}+\rho^{b}$. In the clean limit, $\rho^{b}=\left(\begin{array}{cc}0 & -\frac{h}{e^{2}} \\ \frac{h}{e^{2}} & 0\end{array}\right)$ and $\left|\rho^{f}\right| \ll 1$ is metallic. Then we get $\rho_{x y}^{c} \sim \frac{h}{e^{2}} \gg \rho_{x x}^{c} \sim \rho_{x x}^{f}$. Then the Hall angle $\tan ^{-1}\left(\frac{\rho_{x y}^{c}}{\rho_{x x}^{c}}\right)$ is close to, but strictly smaller, than $90^{\circ}$. More details on this $\mathrm{CFL}$ phase can be found in the Appendixes.

Thus the spin-unpolarized composite Fermi liquid provides a concrete interesting intermediate-coupling metallic state with a large Hall angle. This state will show $\mathrm{SdH}$ oscillations with a frequency that, in contrast to the partially spinpolarized Fermi liquid, matches the band theory Fermi liquid. Other related novel states of matter can also be contemplated, but we will leave their elaboration to the future.

Though the Hall angle in the experiments is large, it clearly does not precisely match the expectation of an ideal quantized anomalous Hall system or of the composite Fermi liquid. This is possibly due both to the presence of disorder and to the presence of the quasiperiodic potential. In particular, the quasiperiodic potential may produce nearly extended in-gap states which may reduce the Hall angle to close to $45^{\circ}$ in the experiment. Finally, we remark that in both the QAH and the CFL state the conduction is predominantly through the sample edge, which will lead to a nonlocal response consistent with experiments.

\section{CORRELATED INSULATOR AT $v=\frac{1}{2}$}

We now turn to the correlated insulator observed at $v=\frac{1}{2}$. While more exotic phases cannot be ruled out, the observed twofold degeneracy of the Landau fan [6] suggests a simple picture of a ferromagnetic insulator. Valley polarization can be ruled out because there are no anomalous Hall signatures in the experiment. For a spin-polarized insulator, the Landau fan from it should have sequence $v=1,2, \ldots$ because of the splitting of the zeroth Landau levels from the two valleys [64]. Especially, the $v=1$ quantum Hall state should have a large charge gap equal to $\Delta$ [64]. However, in the experiment the first Landau fan sequence seen is 2 instead of 1 [6]. Therefore spin polarization may not be the solution at half filling. We suggest the $v=\frac{1}{2}$ insulator has an intervalley-coherent order (IVC) with $\tau_{x, y}$ or $\tau_{x, y} \vec{\sigma}$ valley polarization (see Appendixes). Energetically, within a Hartree-Fock theory in momentum space such an IVC order is known to be favored when the anisotropy $\delta \xi(\mathbf{k})=\left|\xi_{+}(\mathbf{k})-\xi_{-}(\mathbf{k})\right|$ is large $[8,12,59]$. In the Appendixes we show that $\tau_{x} \vec{\sigma}$ is selected by the intervalley Hund's term breaking $\mathrm{U}(2)_{+} \times \mathrm{U}(2)_{-}$symmetry of separate spin and charge conservation of each valley down to $\mathrm{U}(1)_{\text {charge }} \times \mathrm{U}(1)_{\text {valley }} \times \mathrm{SU}(2)_{\text {spin }}$. An in-plane magnetic field $H_{x}$ further favors $\tau_{x} \sigma_{y, z}$. The Appendixes also show that the magnetoresistance for the IVC order can be positive or negative depending on details. Therefore it does not contradict the positive magnetoresistance observed in the experiment [6].

\section{CONCLUSION}

In conclusion, we described several aspects of the physics of magic angle twisted bilayer graphene aligned with a h-BN substrate. The $C_{2}$ breaking due to alignment with h-BN gaps the Dirac points of TBG and further renders the conduction and valence bands with Chern numbers $C= \pm 1$. This suggests a natural explanation of the recent observation [6] of a large anomalous Hall effect at 3/4 filling of the conduction band, as a spontaneously spin-valley polarized ferromagnetic Chern insulator. Energetically, such a state is natural when the 
Coulomb interaction is strong compared to the bandwidth. At intermediate coupling, other more novel states with a large anomalous but unquantized Hall effect are possible. The concrete example we discussed-a spin-unpolarized composite Fermi liquid-may provide an alternate explanation of the data. We constructed a lattice extended Hubbard model for $\mathrm{TBG} / \mathrm{h}-\mathrm{BN}$ which may be useful for future numerical explorations of intermediate coupling phases. Furthermore, this model could also provide an effective model for TBG if $C_{2} T$ is spontaneously broken, which may be also relevant to recent experiments reported in Ref. [65].

Note added in proof. Our discussion overlaps that of a recent paper by N. Bultinck et al., also on TBG/h-BN [66].

\section{ACKNOWLEDGMENTS}

We thank A. Sharpe and D. Goldhaber-Gordon for sharing their data prior to publication and for very useful comments. We thank M. Zaletel for inspiring conversations about this and related problems. This work was supported by NSF Grant No. DMR-1608505 and partially through a Simons Investigator Award from the Simons Foundation to T.S.

\section{APPENDIX A: BAND STRUCTURE CALCULATION}

The continuum model for the TBG is [56]

$$
\begin{aligned}
H_{T B G}= & \sum_{\alpha=t, b} \sum_{\mathbf{k}} \psi_{\alpha}^{\dagger}(\mathbf{k}) h_{0}^{+} \psi_{\alpha}(\mathbf{k}) \\
& +\sum_{\mathbf{k}, j=1,2,3}\left(\psi_{t}^{\dagger}\left(\mathbf{k}+\mathbf{Q}_{\mathbf{j}}\right) T_{j} \psi_{b}(\mathbf{k})+\text { H.c. }\right)
\end{aligned}
$$

Here we focus on valley + . The other valley is related by time-reversal transformation. $\psi_{\alpha}$ is a two-component spinor in terms of the $A$ and $B$ sublattice for each layer. $h_{0}^{\dagger}$ is the standard Dirac Hamiltonian:

$$
h_{0}^{+}=k_{x} \mu_{1}+k_{y} \mu_{2},
$$

where $\mu_{a}$ is the Pauli matrix in the sublattice $A, B$ space for the top layer or the bottom layer. $T_{j}, j=1,2,3$ is the moiré term for the interlayer coupling. $\mathbf{Q}_{\mathbf{1}}=R_{-\theta_{M} / 2} \mathbf{K}_{\mathbf{o}}-R_{\theta_{M} / 2} \mathbf{K}_{\mathbf{o}}$,

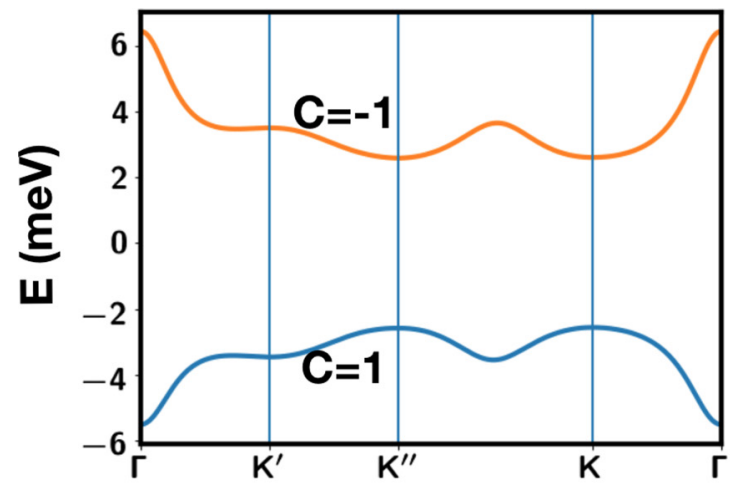

(a) where $R_{\theta}$ rotates a $2 D$ vector by $\theta$ around the $z$ direction. $K_{o}$ is one of the corners of their original large Brillouin zone:

$$
T_{1}=w_{0}-w_{1} \mu_{1},
$$

where $Q_{2}, Q_{3}$ and $T_{2}, T_{3}$ are generated by $C_{3}$ symmetry: $\psi_{\alpha}(\mathbf{k}) \rightarrow e^{i \frac{2 \pi}{3} \mu_{3}} \psi_{\alpha}\left(C_{3} \mathbf{k}\right)$.

We use the parameters $w_{1}=110 \mathrm{meV}$ and $\frac{w_{0}}{w_{1}}=0.7$ [13] to incorporate the lattice relaxation effects. This value of $\frac{w_{0}}{w_{1}}$ gives a hybridization gap of around $30-40 \mathrm{meV}$ between the valence band and the band below for twist angle $\theta_{M}=1.05^{\circ}-1.20^{\circ}$, consistent with the experimental measurement [2].

As argued in the main text, the aligned h-BN layer on the top provides a mass term for the top graphene layer:

$$
H_{h B N}=M \sum_{\mathbf{k}} \psi_{t}^{\dagger}(\mathbf{k}) \mu_{z} \psi_{t}(\mathbf{k}) \text {. }
$$

We then diagonalize $H_{T B G}+H_{h B N}$ from Eqs. (A1) and (A4) and get the band structure shown in Fig. 1 of the main text. Due to the twist angle $\theta_{M}$, the original Dirac cone at $K_{o}$ point for the top graphene layer is put at the $K^{\prime}$ point of the mini Brillouin zone (MBZ) while the original Dirac cone at $K_{o}$ for the bottom graphene layer is put at the $K$ point of the MBZ.

In the presence of the $M$ term in the Eq. (A4), the Dirac crossing is gapped at the $K^{\prime}$ point even if we suppress the interlayer coupling $T_{j}$ to zero. With the interlayer coupling $T_{j}$, the Dirac cone at the $K$ point is also gapped. The band gap at $K^{\prime}$ is around $10 \mathrm{meV}$ while the band gap at the $K$ point is only $5 \mathrm{meV}$. Note that there is no symmetry relating $K$ and $K^{\prime}$. Also, the conduction and the valence bands are well separated from the other bands using $\frac{w_{0}}{w_{1}}=0.7$, as shown in Fig. 3, and from each other. Thus the Chern numbers are well defined for both conduction and valence bands. We calculate the Chern number of each band following the same method used in Ref. [8]. For the valley + , we find that the conduction and the valence bands have Chern numbers $C=1$ and $C=-1$, respectively. Because of the timereversal symmetry, the other valley must have opposite Chern numbers.

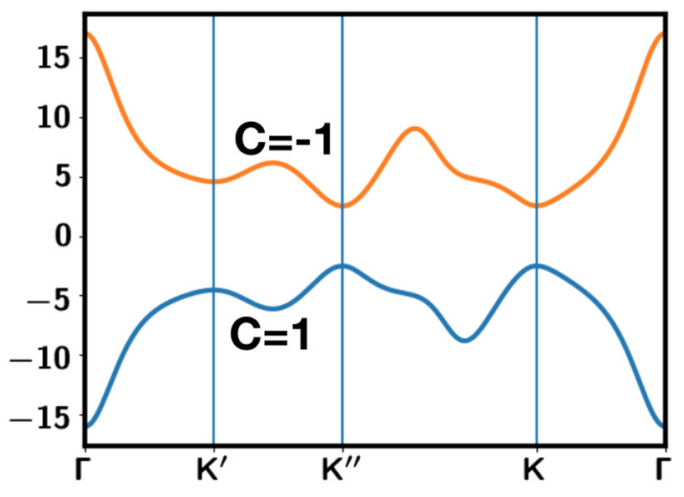

(b)

FIG. 3. Band structure for valley + of the TBG-h-BN system in the MBZ: (a) $\theta=1.08^{\circ}$ and (b) $\theta=1.20^{\circ}$. The middle two bands are well separated from the other bands. The middle two bands have Chern numbers $C=1$ and $C=-1$. We use $\frac{w_{0}}{w_{1}}=0.7$ to incorporate the lattice relaxation effects. 
TABLE I. Tight-binding model parameters for the modified Haldane model in units of meV.

\begin{tabular}{|c|c|c|c|c|c|c|c|}
\hline$\theta_{M}$ & $m_{0}$ & $t$ & $t_{A}^{\prime}$ & $t_{B}^{\prime}$ & $t^{\prime \prime}$ & $t_{1}^{\prime \prime \prime}$ & $t_{2}^{\prime \prime \prime}$ \\
\hline $1.08^{\circ}$ & 1.36 & 1.22 & $0.670 e^{i 0.366 \pi}$ & $0.731 e^{-i 0.657 \pi}$ & $0.801 e^{-i 0.685 \pi}$ & $0.123 e^{-i 0.48 \pi}$ & $0.355 e^{-i 0.411 \pi}$ \\
\hline $1.20^{\circ}$ & 0.076 & 3.056 & $0.837 e^{i 0.56 \pi}$ & $0.828 e^{-i 0.469 \pi}$ & $2.062 e^{-i 0.54 \pi}$ & $0.915 e^{-i 0.337 \pi}$ & $0.815 e^{-i 0.434 \pi}$ \\
\hline
\end{tabular}

\section{Valley Zeeman coupling}

As discussed in the main text, the alignment of the h-BN gives a mass to the Dirac cones at the $K$ and $K^{\prime}$ points in the MBZ for each spin and each valley. As is well known, a massive Dirac fermion can have an out-of-plane magnetic moment, which is opposite for opposite valleys. Therefore there is a valley Zeeman coupling to the out-of-plane magnetic field:

$$
H_{v}=-\frac{1}{2} \mu_{B} B_{z} \sum_{\mathbf{k}} g_{v}(\mathbf{k}) c^{\dagger}(\mathbf{k}) \tau_{z} c(\mathbf{k}),
$$

with $g_{v} \sim \frac{1}{\Delta}$. We numerically calculated $g_{v}$ for $\theta_{M}=1.20^{\circ}$ and $M=15 \mathrm{meV}$. Indeed, we find that close to the $K$ and $K^{\prime}$ point, $g_{v}(K) \approx g_{v}\left(K^{\prime}\right) \approx 15$ for both the conduction and valence bands, which is 1 order of magnitude larger than the spin Zeeman coupling. Averaging over the whole MBZ, we have $\bar{g}_{v} \approx 4$ because the valley Zeeman coupling away from the $K$ and $K^{\prime}$ points is small.

We need to emphasize that the valley Zeeman coupling does not capture all effects of an out-of-plane magnetic field. We should also include the part corresponding to the Landau levels of a nonrelativistic band without any Berry curvature. Besides, the semiclassical picture on which the valley Zeeman coupling relies is limited to an infinitesimal magnetic field. In graphene moiré superlattice, the magnetic flux is large ( $\frac{1}{6}$ of flux quanta) for $B_{z} \approx 5$ Tesla in the experiment. Therefore, the best way to capture the response to $B_{z}$ is to calculate the Landau levels directly. This approach has been taken by Ref. [64]. At the neutrality point, the low-energy physics is captured by one massive Dirac cone per valley. Because of the polarization of pseudospin for the zeroth Landau level of a Dirac cone, the zeroth Landau levels from the two valleys are split by a gap equal to $\Delta$. Therefore we expect a Landau fan with $v=2,4$ at neutrality where the twofold degeneracy is from the spin. This is indeed what has been observed in the experiment [6].

\section{APPENDIX B: LATTICE MODEL}

The TBG system has a Wannier obstruction to construct a valley-preserving and $C_{2} T$ symmetric model $[12,36,37,48,49]$. In the TBG-hBN, the $C_{2} T$ is broken by the alignment to h-BN. As a result, there is no Wannier obstruction for a valley-preserving lattice model. For each valley, where the conduction and the valence bands have the opposite Chern numbers, we can build a lattice model by combining both bands. We constructed the Wannier orbitals following the standard projection methods [67]. The resulting lattice model is a modified "Haldane model" on a honeycomb lattice for each valley. However, the two lattice sublattice sites correspond to the $A B$ and $B A$ regions, while the density is concentrated on the $A A$ regions. Therefore each Wannier orbital has the shape of a fidget spinner, similar to Ref. [12].

\section{Tight-binding parameters}

In Table I we show tight-binding parameters defined in the main text for two twist angles.

\section{Quasiperiodic potential}

We also need to add a quasiperiodic potential term from projecting the $V_{j}$ term in Eq. (2) to the two orbitals:

$$
H_{Q P}=V_{Q P} \sum_{i} \cos \left(\mathbf{Q}_{j}^{\prime} \cdot \mathbf{R}_{\mathbf{i}}\right) c_{i ; a \sigma}^{\dagger} c_{i ; a \sigma},
$$

where we ignored the quasiperiodicity in the hopping terms for simplicity. The value of $V_{Q P}$ can be tuned by the displacement field, and we keep it as a free parameter.

\section{3. $M \rightarrow 0$ limit}

For any finite $M$ we can build a lattice model on a honeycomb lattice, as done in the main text. This process can even be extrapolated to the $M \rightarrow 0$ limit. How is this consistent with the Wannier obstruction at $M=0$ ? In the following we try to resolve this puzzle. The Wannier obstruction at $M=0$ is related to the $C_{2} T$ symmetry and is therefore different from the intrinsic Wannier obstruction for the Chern band. Once $C_{2} T$ breaking is allowed, an exponentially localized Wannier orbital is possible and we can actually recover the gapless Dirac crossing with a finite range of hopping, such as $R<7$. This process is conceptually similar to the Wannier construction process by ignoring the $\mathrm{U}(1)$ valley symmetry in Ref. [12].

In this process, the lattice model contains $C_{2} T$ breaking terms even in the $M \rightarrow 0$ limit. The typical $C_{2} T$ breaking term is the next-nearest-neighbor hopping $t_{A}^{\prime} \approx-t_{B}^{\prime}=t^{\prime}$. As shown in Fig. 4, when the external $C_{2} T$ breaking term $M$ is large, the lattice model has a $C_{2} T$ breaking term $t^{\prime} \propto M$. When $M$ is small, the $C_{2} T$ breaking term in our lattice model is obviously overestimated. With enough range of hopping, we can still reduce the band gap in the lattice model to be proportional to $M$ at the $M \rightarrow 0$ limit. This means that the lattice model with enough range of hopping has a hidden nonlocal $C_{2} T$ symmetry. If one can keep all these nonlocal terms in the lattice model, one can still get the correct result of Dirac crossings between the valence and conduction bands. However, the purpose of a lattice model is to do a useful approximation. Such a lattice model with a nonlocal $C_{2} T$ symmetry (see, e.g., Ref. [60]) is dangerous because it is not clear how to do approximate calculations that maintain this symmetry. (Similarly, if one does not insist on a local representation of the $\mathrm{U}(1)_{\text {valley }}$ symmetry, a lattice model is also possible in the $M \rightarrow 0$ limit, as explicitly done in Ref. [12]). 


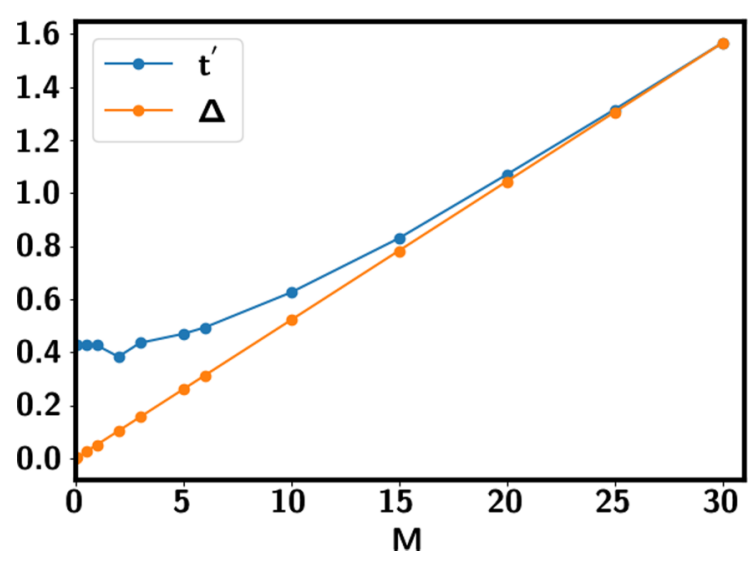

FIG. 4. The $C_{2} T$ breaking term in the lattice model $t^{\prime}$ and the band gap $\Delta$ with $M$. As $M \rightarrow 0$, the lattice model does not have obvious $C_{2} T$ symmetry.

Thus a useful lattice model that keeps just the states from the active bands is possible only for the large- $M$ regime, i.e., when $\mathrm{h}-\mathrm{BN}$ is aligned.

\section{APPENDIX C: FERROMAGNETIC INSULATOR AT $v=\frac{1}{2}$}

At $v=\frac{1}{2}$, there is exactly one hole at each honeycomb lattice site. Therefore a strong-coupling approach for $U \gg$ $t, t^{\prime}$ with an intersite Hund's term favors a simple insulator which puts a spin-valley polarized hole at each site $[59,60]$. Either $\tau_{z}$ or $\tau_{x}$ valley polarization is selected, depending on the competition between the interaction and the kinetic term [59]. However, this phase leaves both the conduction and valence band of one flavor empty, which costs kinetic energy. Therefore, for the experimentally relevant $U \sim W$ regime, the strong-coupling approach is also not appropriate at $v=\frac{1}{2}$.

For $U \sim W$, a more natural ferromagnetic (FM) order is one where only the conduction bands of two flavors are pushed up while the other two conduction bands are fully filled. This is described by the order parameter $c^{\dagger} \tau_{a} \sigma_{b} c$, where $c$ is the electron destruction operator for the conduction band (we suppressed the spin-valley index). $\tau^{a}$ are Pauli matrices in valley space, and $\sigma^{a}$ are spin Pauli matrices.

We consider only FM order without momentum dependence (i.e., the particle-hole pair that forms the order parameter has zero internal momentum). There are 15 such order parameters corresponding to $\tau^{a}, \sigma^{a}, \tau^{a} \sigma^{b}$. Our system has an approximate symmetry $\mathrm{U}(2)_{+} \times \mathrm{U}(2)_{-}[8,12]$ generated by $\vec{\sigma}, \tau_{z} \vec{\sigma}, \tau_{z}$ and the total charge. For any particlehole order $\psi^{\dagger} A \psi$ with $A$ a $4 \times 4$ matrix, another order is degenerate from the spin-valley rotation: $A \rightarrow U A U^{\dagger}$ where $U \in \mathrm{U}(2)_{+} \times \mathrm{U}(2)_{-}$. It is then easy to verify that the $15 \mathrm{FM}$ orders can be grouped to three classes: (1) $\tau_{z}$; (2) $\tau_{z} \vec{\sigma}, \vec{\sigma}$; and (3) $\tau_{x, y}, \tau_{x, y} \vec{\sigma}$. We can try to decide which of these distinct FM orders is selected by the anisotropies in the Hamiltonian based on a simple Hartree-Fock calculation. For fully polarized states with $\tau^{z}$ or $\tau^{z} \vec{\sigma}$ ordering, the Hartree-Fock energies are readily seen to be the same. Thus it suffices to compare $\tau^{z}$ and $\tau^{x, y}$ ordering. As argued in previous papers $[8,12]$, within such a Hartree-Fock calculation, either $\tau^{z}$ or $\tau^{x, y}$ ordering wins depending on the bandwidth. The flat-band limit prefers $\tau^{z}$, while for wider bands it is possible to stabilize $\tau^{x, y}$. A further selection within each group of orders related by $\mathrm{U}(2)_{+} \times \mathrm{U}(2)_{-}$occurs through a weak intervalley Hund's interaction that locks spins in the two valleys together.

We therefore proceed phenomenologically and ask which such order is consistent with results from experiments [6] at $v=\frac{1}{2}$. First, $\tau_{z}$ can be ruled out because of the absence of the anomalous Hall effect at this filling. Next we discuss the spin polarization $\sigma_{z}$ order. For a spin-polarized insulator, we expect that the Landau fan degeneracy from it is one-half of the one at neutrality. Therefore the Landau fan expected should be $v=$ $1,2, \ldots$ Besides, the quantum Hall sequence at $v=1$ should have a charge gap $\propto$ to $\Delta$ [64]. However, in the experiment the degeneracy of the first Landau fan observed is 2 instead of 1. This implies that a simple $\sigma_{z}$ order may not work.

In contrast, $\tau_{x, y}$ or $\tau_{x, y} \vec{\sigma}$ order is consistent with the twofold Landau fan degeneracy because there is still spin. In the next section we discuss the IVC order using the lattice Haldane model. Before we proceed, we need to first decide which one among $\tau_{x}$ and $\tau_{x} \vec{\sigma}$ is selected by the intervalley Hund's term. In the lattice model, the dominant intervalley Hund's term can be written as an on-site four-fermion interaction [59]:

$$
H_{J}=g \sum_{i} \sum_{\sigma_{1}, \sigma_{2}=\uparrow, \downarrow} c_{+\sigma_{1}}^{\dagger}(i) c_{-\sigma_{2}}^{\dagger}(i) c_{+\sigma_{2}}(i) c_{-\sigma_{1}}(i) .
$$

Note that $g>0$ corresponds to intervalley Hund's while $g<0$ corresponds to anti-Hund's. We only need to compare the expectation value of $H_{J}$ for $\tau_{x}$ and $\tau_{x} \sigma_{z}$ order. Using Wick's theorem,

$$
\begin{aligned}
H_{J}= & -g \sum_{i} \sum_{\sigma_{1}, \sigma_{2}=\uparrow, \downarrow}\left\langle c_{+\sigma_{1}}^{\dagger}(i) c_{+\sigma_{2}}(i)\right\rangle\left\langle c_{-\sigma_{2}}^{\dagger}(i) c_{-\sigma_{1}}(i)\right\rangle \\
& +g \sum_{i} \sum_{\sigma_{1}, \sigma_{2}=\uparrow, \downarrow}\left\langle c_{+\sigma_{1}}^{\dagger}(i) c_{-\sigma_{1}}(i)\right\rangle\left\langle c_{-\sigma_{2}}^{\dagger}(i) c_{+\sigma_{2}}(i)\right\rangle .
\end{aligned}
$$

It is easier to work in the $\tau_{x}$ basis instead of $\tau_{z}$. We label $\mathrm{S}$ and $\mathrm{A}$ as the valley polarization corresponding to 1 and -1 of $\tau_{x}$. We have $c_{ \pm, \sigma}(i)=\frac{1}{\sqrt{2}}\left[c_{S \sigma}(i) \pm c_{A \sigma}(i)\right]$. Under time reversal, $c_{S} \rightarrow c_{S}$ while $c_{A} \rightarrow-c_{A}$. Thus for a time-reversal invariant ansatz, $\left\langle c_{S \sigma}^{\dagger}(i) c_{A \sigma}(i)\right\rangle=$ 0 . This leads to $\left\langle c_{+\sigma}^{\dagger}(i) c_{+\sigma}(i)\right\rangle=\left\langle c_{-\sigma}^{\dagger}(i) c_{-\sigma}(i)\right\rangle=$ $\frac{1}{2}\left(\left\langle c_{S \sigma}^{\dagger}(i) c_{S \sigma}(i)\right\rangle+\left\langle c_{A \sigma}^{\dagger}(i) c_{A \sigma}(i)\right\rangle\right) \quad$ and $\quad\left\langle c_{+\sigma}^{\dagger}(i) c_{-\sigma}(i)\right\rangle=$ $\left\langle c_{-\sigma}^{\dagger}(i) c_{+\sigma}(i)\right\rangle=\frac{1}{2}\left(\left\langle c_{S \sigma}^{\dagger}(i) c_{S \sigma}(i)\right\rangle-\left\langle c_{A \sigma}^{\dagger}(i) c_{A \sigma}(i)\right\rangle\right)$.

We label $n_{p, \sigma}(i)=\frac{1}{2}\left\langle c_{S \sigma}^{\dagger}(i) c_{S \sigma}(i)+c_{A \sigma}^{\dagger} c_{A \sigma}(i)\right\rangle \quad$ and $n_{m, \sigma}(i)=\frac{1}{2}\left\langle c_{S \sigma}^{\dagger}(i) c_{S \sigma}(i)-c_{A \sigma}^{\dagger}(i) c_{A \sigma}(i)\right\rangle$. Because both $\tau_{x}$ and $\tau_{x} \sigma_{z}$ commute with $\sigma_{z}$, we know that $\left\langle c_{+\sigma_{1}}^{\dagger}(i) c_{+\sigma_{2}}(i)\right\rangle$ is nonzero only for $\sigma_{1}=\sigma_{2}$. Therefore,

$$
\begin{aligned}
\left\langle H_{J}\right\rangle= & -g \sum_{\sigma} \sum_{i}\left\langle c_{+\sigma}^{\dagger}(i) c_{+\sigma}(i)\right\rangle\left\langle c_{-\sigma}^{\dagger}(i) c_{-\sigma}(i)\right\rangle \\
& +g \sum_{\sigma_{1} \sigma_{2}} \sum_{i}\left\langle c_{+\sigma_{1}}^{\dagger}(i) c_{-\sigma_{1}}(i)\right\rangle\left\langle c_{-\sigma_{2}}^{\dagger}(i) c_{+\sigma_{2}}(i)\right\rangle \\
= & -g \sum_{\sigma} \sum_{i} n_{p \sigma}(i) n_{p \sigma}(i)+g \sum_{\sigma_{1} \sigma_{2}} \sum_{i} n_{m \sigma_{1}}(i) n_{m \sigma_{2}}(i) \\
= & -g \sum_{\sigma} \sum_{i} n_{p \sigma}(i) n_{p \sigma}(i)+g \sum_{i} n_{m}(i) n_{m}(i),
\end{aligned}
$$

where $n_{m}(\mathbf{k})=n_{m \uparrow}(\mathbf{k})+n_{m \downarrow}(\mathbf{k})$ is the difference between the occupation number of the $S$ band and the $A$ band. 


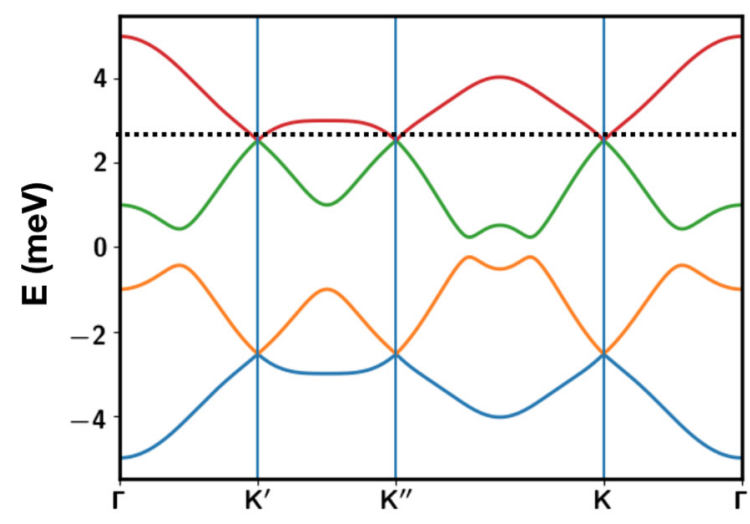

(a) $M=0$

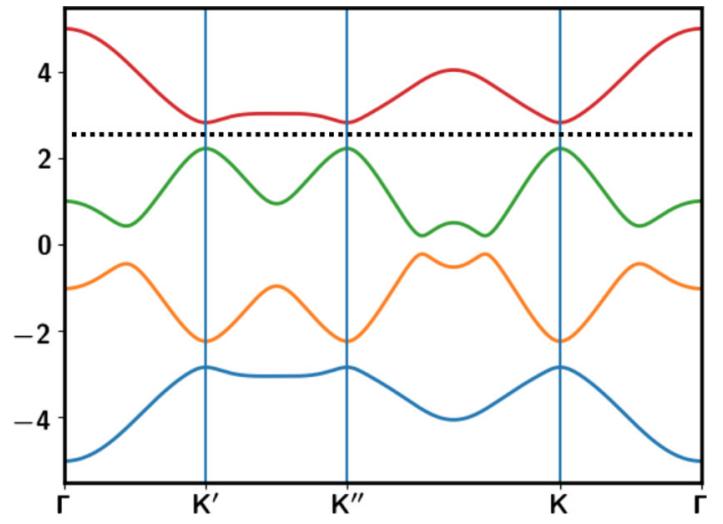

(b) $M=0.3$

FIG. 5. Band structures for IVC order with (a) $M=0$ and (b) $M=0.3$. We use $t=1, t^{\prime}=0.3 i$, and $\Phi=2$. Dashed line labels the half filling of the conduction band.

For $\tau_{x}$ and $\tau_{x} \sigma_{z}$ order, the first term is the same because $n_{p \sigma}(i)$ is basically the density for the spin $\sigma$, which is fixed by the total density. To minimize the second term, we need to minimize $n_{m}(i)$. For $\tau_{x}$ order, $n_{m \uparrow}(i)=n_{m \downarrow}(i)$, while for $\tau_{x} \sigma_{z}$ order we have $n_{m \uparrow}(i)=-n_{m \downarrow}(i)$. Therefore we conclude that $\tau_{x} \vec{\sigma}$ is selected by the intervalley Hund's term $g>0$.

$$
h_{+}(\mathbf{k})=\left(\begin{array}{c}
M-2 t^{\prime}\left[\cos \left(k_{y}+\varphi\right)+2 \cos \left(\frac{\sqrt{3}}{2} k_{x}\right) \cos \left(\frac{1}{2} k_{y}-\varphi\right)\right] \\
-t\left(e^{-i \frac{k_{x}}{\sqrt{3}}}+2 e^{i \frac{k_{x}}{2 \sqrt{3}}} \cos \frac{k_{y}}{2}\right)
\end{array}\right.
$$

for valley + , and

$$
h_{-}(\mathbf{k})=\left(\begin{array}{c}
M-2 t^{\prime}\left[\cos \left(-k_{y}+\varphi\right)+2 \cos \left(\frac{\sqrt{3}}{2} k_{x}\right) \cos \left(-\frac{1}{2} k_{y}-\varphi\right)\right] \\
-t\left(e^{-i \frac{k_{x}}{\sqrt{3}}}+2 e^{i \frac{k_{x}}{2 \sqrt{3}}} \cos \frac{k_{y}}{2}\right)
\end{array}\right.
$$

for valley -

Next we add IVC order. First let us add $\tau_{x}$ order to avoid the complexity from spin. Generalization to $\tau_{x} \sigma_{z}$ order is straightforward. The mean-field ansatz we add is simply

$$
H_{I V C}=\Phi \sum_{\sigma} \sum_{i} c_{+\sigma}^{\dagger}(i) c_{-\sigma}(i)+c_{-\sigma}^{\dagger}(i) c_{+\sigma}(i)
$$

In Fig. 5 we show the band structures after adding IVC for $m_{0}=0$ and $m_{0} \neq 0$ cases separately. For $M=0$ we find there is Dirac crossing at half filling. This is just a manifestation of existence of nodes of IVC orders in momentum space for \pm Chern bands, as pointed out by Ref. [66]. Basically, if we project the IVC order parameter in Eq. (C6) to the conduction band, the corresponding IVC order in momentum space $\tilde{\Phi}(\mathbf{k})=\Phi \mu_{+}^{*}(\mathbf{k}) \mu_{-}(\mathbf{k})$ inherits the winding of Bloch wave functions. Because $\mu_{+}(\mathbf{k})$ has a $2 \pi$ winding while $\mu_{-}(\mathbf{k})$ has a $-2 \pi$ winding in the MBZ, $\tilde{\Phi}(\mathbf{k})$ inherits a $-4 \pi$ winding in the MBZ. This means there are two vortices associated with the complex field $\tilde{\Phi}(\mathbf{k})$. In terms of conduction band, we have
In the following part we discuss the property of the IVC order $\tau_{x}$. For simplicity, we only keep the following tightbinding parameters: $m_{0}=M, t$ and $t_{A}^{\prime}=t_{B}^{\prime *}=t^{\prime}$. Note this choice is just the standard Haldane model for which $t_{A}^{\prime}=t_{B}^{\prime *}$. In this simple model, the $M=0$ limit has inversion symmetry.

In momentum space, we have

$$
\left.\begin{array}{c}
-t\left(e^{i \frac{k_{x}}{\sqrt{3}}}+2 e^{-i \frac{k_{x}}{2 \sqrt{3}}} \cos \frac{k_{y}}{2}\right) \\
-M-2 t^{\prime}\left[\cos \left(k_{y}-\varphi\right)+2 \cos \left(\frac{\sqrt{3}}{2} k_{x}\right) \cos \left(\frac{1}{2} k_{y}+\varphi\right)\right]
\end{array}\right)
$$

$$
\left.\begin{array}{c}
-t\left(e^{i \frac{k_{x}}{\sqrt{3}}}+2 e^{-i \frac{k_{x}}{2 \sqrt{3}}} \cos \frac{k_{y}}{2}\right) \\
-M-2 t^{\prime}\left[\cos \left(-k_{y}-\varphi\right)+2 \cos \left(\frac{\sqrt{3}}{2} k_{x}\right) \cos \left(-\frac{1}{2} k_{y}+\varphi\right)\right]
\end{array}\right)
$$

the mean-field ansatz

$$
H_{M}(\mathbf{k})=A_{x}(\mathbf{k}) \tau_{x}+A_{y}(\mathbf{k}) \tau_{y}+A_{z}(\mathbf{k}) \tau_{z},
$$

because $A_{x}(\mathbf{k})=\operatorname{Re} \tilde{\Phi}(\mathbf{k})$ and $A_{y}(\mathbf{k})=\operatorname{Im} \tilde{\Phi}(\mathbf{k}) \cdot \tau_{\mu}$ are Pauli matrices in the valley space, and $A_{x}$ and $A_{y}$ are from IVC orders. We conclude that the vector $\left(A_{x}, A_{y}\right)$ has two vortices in the MBZ. Because of the time-reversal symmetry and $C_{3}$ rotation symmetry, these two vortices are pinned at the $K$ and $K^{\prime}$ point. Close to the $K$ point, we have $\left(A_{x}, A_{y}\right)=v_{F}\left(k_{x}, k_{y}\right)$. When $M=0$, there is still inversion symmetry within each valley. Therefore $A_{z}(\mathbf{k})=0$ and we have two Dirac crossings. In the real TG-hBN problems, there is, of course, no inversion symmetry within each valley. So at each momentum the two valleys have different energies and generically $A_{z}(\mathbf{k}) \neq 0$. Therefore one gets a gap as shown in Fig. 5.

Because of time-reversal symmetry, there is degeneracy between $K$ and $K^{\prime}$. Naively, we expect fourfold degeneracy in Landau levels from the IVC insulator. However, as pointed out in Ref. [64], $C_{3}$ breaking will reduce the mini valley degeneracy. In the TBG samples, scanning tunneling 
microscopy experiments show quite significant $C_{3}$ breaking. Therefore the IVC order is consistent with the twofold Landau fan degeneracy in the experiment [6].

In the experiment [6], the resistance for the correlated insulator at half filling increases under the out-of-plane magnetic field $B_{z}$. Next we show that this positive magnetoresistance is also consistent with IVC order. As shown in Fig. 5, the charge gap is mainly decided by the band gap at $K$ and $K^{\prime}$ in the MBZ. Therefore we can write down a low-energy mean-field model close to the $K$ and $K^{\prime}$ points by assuming IVC order:

$$
h_{K}(\mathbf{k})=v_{F} k_{x} \tau_{x}+v_{F} k_{y} \tau_{y}+\left[M+A\left(k_{x}^{2}+k_{y}^{2}\right)\right] \tau_{z},
$$

where $\tau_{x}, \tau_{y}$ terms are from IVC order.

By time-reversal symmetry $\tau_{x}$, the low-energy model at $K^{\prime}$ is fixed to be

$$
h_{K^{\prime}}(\mathbf{k})=-v_{F} k_{x} \tau_{x}-v_{F} k_{y} \tau_{y}-\left[M+A\left(k_{x}^{2}+k_{y}^{2}\right)\right] \tau_{z} .
$$

Under $B_{z}$, the Landau levels of these two massive Dirac cones can be solved exactly. The zeroth Landau levels at $K$ and $K^{\prime}$ have energies

$$
E_{0}^{K}\left(B_{z}\right)=M+2 A B_{z}, \quad E_{0}^{K^{\prime}}\left(B_{z}\right)=-M-2 A B_{z} .
$$

Therefore the charge gap for the $v=1 / 2$ correlated insulator is

$$
\Delta_{c}\left(B_{z}\right)=2 M+4 A B_{z} .
$$

If $A>0$, there is a positive magnetoresistance. Microscopically, $\left[M+A\left(k_{x}^{2}+k_{y}^{2}\right)\right] \tau_{z}$ is just the energy difference between the two valleys. A nonzero $A$ can be generated by adding $t_{A}^{\prime} \neq t_{B}^{\prime}$. The sign of $A$ can be arbitrary depending on details. The magnitude can be large if $t_{A}^{\prime}-t_{B}^{\prime}$ is large. In conclusion, the positive magnetoresistance is consistent with the IVC order. In the above we use $\tau_{x}$ order, and the same conclusion holds for $\tau_{x} \sigma_{z}$ order.

The response to the in-plane magnetic field $H_{x}$ (assuming it couples predominantly to the spin), however, depends on whether $\tau_{x}$ or $\tau_{x} \vec{\sigma}$ is selected. Such a field can further split the energy degeneracy among $\tau_{x} \vec{\sigma}$. Because there is no spin magnetization, the field energy at first order of perturbation vanishes. For the second-order perturbation, $\tau_{x} \sigma_{y, z}$ order can have a negative energy correction because $\tau_{x} \sigma_{y, z}$ anticommutes with $\sigma_{x}$. Therefore the in-plane magnetic field favors $\tau_{x} \sigma_{y, z}$. The splitting is of order $\frac{g_{s}^{2} \mu_{B}^{2} H_{x}^{2}}{\Phi}$ and therefore is small.

\section{APPENDIX D: SPINFUL COMPOSITE FERMION LIQUID}

We give theoretical descriptions of several spinful CFL phases for the filling $\nu_{T}=\frac{1}{2}+\frac{1}{2}$ of the spinful $C=1$ Chern band. As discussed in the main text, in the strict flat-band limit, the simple ferromagnetic insulator will win. But the states discussed in this Appendix may be competitive once band dispersion becomes significant, i.e., for intermediate coupling $U \sim W$.

We do a slave-boson parton construction $c_{i ; \sigma}=b f_{i ; \sigma}$. (We can also do a slave-fermion parton, which leads to a "quantum Hall spin liquid" insulator mentioned briefly at the end of this section.) $b$ is a spinless boson which carries the physical charge while $f_{\sigma}$ is a neutral spin- $1 / 2$ fermion. We have filling $n_{b}=1$ and $\sum_{\sigma} n_{f ; \sigma}=1$. Besides, $b$ and $f$ need to couple to an internal $U(1)$ gauge field $a$ with opposite charges.
In this parton construction we can access different phases by putting $b$ and $f$ in different phases. For the fermion $f$, the most natural ansatz is just a spin-unpolarized state with a Fermi surface for each spin component. The spinless boson at $v=1$ of a $C=1$ Chern band can be either a Pfaffian state or itself form a composite Fermi-liquid phase. For simplicity and because it is somewhat more familiar, here we focus on the former case. Then the boson has a quantum Hall effect with $\sigma_{x y}^{b}=\frac{e^{2}}{h}$. Such a phase has Ising anyons, and the low-energy effective theory is denoted $\left[\mathrm{U}(1)_{4} \times\right.$ Ising) $\left./ Z_{2}\right]$. In our case we need to further couple $b$ to the internal gauge field $a$. For the purpose of the charge response of the microscopic electron, we can ignore the non-Abelian Ising part and just write down the response of the slave boson $b$ to the gauge field $A-$ $a$ it couples to ( $A$ is the external probe electromagnetic gauge field). This is just a Chern-Simons term $\frac{1}{4 \pi}(A-a) d(A-a)$. The low-energy theory for the microscopic electron $c$ is

$$
L=\sum_{\sigma} L\left[f_{\sigma}, a\right]+\frac{1}{4 \pi} a d a-\frac{1}{2 \pi} A d a+\frac{1}{4 \pi} A d A+\ldots
$$

This action resembles that of the standard Halperin-LeeRead theory for the half-filled Landau level [68]. However, for this state other terms need to be included to describe the Ising anyon of the slave boson, though we will not explicitly write them here. For discussing low-energy electrical transport the action above, which describes the Fermi surfaces and the gauge field $a$, is sufficient. In this sense, this CFL phase should have essentially the same properties as the conventional CFL phase. Close to the edge, however, a neutral Majorana mode may be present, unlike the conventional composite Fermi liquid. Besides, the single electron in this CFL phase is gapped because the single-electron operator is a combination of monopole operator and the Ising anyon. This is different from the traditional HLR phase.

From the Ioffe-Larkin rule [63], the resistivity tensor of the original electron is

$$
\rho^{c}=\rho^{f}+\rho^{b}
$$

Therefore we have

$$
\rho^{c}=\rho^{f}+\left(\begin{array}{cc}
0 & -\frac{h}{e^{2}} \\
\frac{h}{e^{2}} & 0
\end{array}\right) .
$$

In the clean limit, $\rho^{f}$ behaves like a metal and thus $\left|\rho^{f}\right| \ll \frac{h}{e^{2}}$. Therefore $\rho_{x y}^{c} \approx \frac{h}{e^{2}} \gg \rho_{x x}^{c}=\rho_{x x}^{f}$. Thus this phase has a large Hall angle, together with nonzero bulk dissipation.

Finally, we point out that the above CFL phase can go through a continuous phase transition by pairing of the composite fermions. In the simplest case we consider a spin singlet pairing $\left\langle f_{\uparrow}^{\dagger} f_{\downarrow}^{\dagger}\right\rangle \neq 0$, and the resulting phase is an insulator with Hall conductivity $\sigma_{x y}=\frac{e^{2}}{h}$. The charge response is actually the same as the spin-polarized Chern insulator. However, in this insulator the spin is in a singlet phase, and the elementary spin excitations are gapped spinons carrying spin $1 / 2$, just like a $Z_{2}$ spin liquid. We dub this exotic insulator as a "quantum Hall spin liquid." It is a nontrivial non-Abelian topological ordered phase. For example, the "vison" excitation in a conventional $Z_{2}$ spin liquid now carries 
$1 / 2$ charge and is an Ising anyon, though it still has $\pi$ mutual statistics with the gapped spinon. Details of this and other "quantum Hall spin liquid" phases will be discussed elsewhere.
[1] E. M. Spanton, A. A. Zibrov, H. Zhou, T. Taniguchi, K. Watanabe, M. P. Zaletel, and A. F. Young, Science 360, 62 (2018).

[2] Y. Cao, V. Fatemi, A. Demir, S. Fang, S. L. Tomarken, J. Y. Luo, J. D. Sanchez-Yamagishi, K. Watanabe, T. Taniguchi, E. Kaxiras et al., Nature (London) 556, 80 (2018).

[3] Y. Cao, V. Fatemi, S. Fang, K. Watanabe, T. Taniguchi, E. Kaxiras, and P. Jarillo-Herrero, Nature (London) 556, 43 (2018).

[4] G. Chen, L. Jiang, S. Wu, B. Lyu, H. Li, B. L. Chittari, K. Watanabe, T. Taniguchi, Z. Shi, J. Jung, Y. Zhang, and F. Wang, Nat. Phys. 15, 237 (2019).

[5] M. Yankowitz, S. Chen, H. Polshyn, K. Watanabe, T. Taniguchi, D. Graf, A. F. Young, and C. R. Dean, Science, 363, 1059 (2019).

[6] A. L. Sharpe, E. J. Fox, A. W. Barnard, J. Finney, K. Watanabe, T. Taniguchi, M. A. Kastner, and D. GoldhaberGordon, Science 365, 605 (2019).

[7] In the presence of $C_{2} T$ symmetry, the Berry phase around a $k$ space loop is fixed to be either 0 or $\pi$. Therefore gapping out of the Dirac crossing is forbidden.

[8] Y.-H. Zhang, D. Mao, Y. Cao, P. Jarillo-Herrero, and T. Senthil, Phys. Rev. B 99, 075127 (2019).

[9] M. Xie and A. H. MacDonald, arXiv:1812.04213.

[10] J. Jung, A. Raoux, Z. Qiao, and A. H. MacDonald, Phys. Rev. B 89, 205414 (2014).

[11] C. Xu and L. Balents, Phys. Rev. Lett. 121, 087001 (2018).

[12] H. C. Po, L. Zou, A. Vishwanath, and T. Senthil, Phys. Rev. X 8, 031089 (2018).

[13] M. Koshino, N. F. Q. Yuan, T. Koretsune, M. Ochi, K. Kuroki, and L. Fu, Phys. Rev. X 8, 031087 (2018).

[14] J. Kang and O. Vafek, Phys. Rev. X 8, 031088 (2018).

[15] N. F. Yuan and L. Fu, Phys. Rev. B 98, 045103 (2018).

[16] J. F. Dodaro, S. A. Kivelson, Y. Schattner, X.-Q. Sun, and C. Wang, Phys. Rev. B 98, 075154 (2018).

[17] M. Ochi, M. Koshino, and K. Kuroki, Phys. Rev. B 98, 081102 (2018).

[18] H. Isobe, N. F. Q. Yuan, and L. Fu, Phys. Rev. X 8, 041041 (2018).

[19] B. Padhi, C. Setty, and P. W. Phillips, Nano Lett. 18, 6175 (2018).

[20] J. M. Pizarro, M. J. Calderón, and E. Bascones, arXiv: 1805.07303

[21] J. Gonzalez and T. Stauber, Phys. Rev. Lett. 122, 026801 (2019).

[22] A. Thomson, S. Chatterjee, S. Sachdev, and M. S. Scheurer, Phys. Rev. B 98, 075109 (2018).

[23] Y. Sherkunov and J. J. Betouras, Phys. Rev. B 98, 205151 (2018).

[24] T. Huang, L. Zhang, and T. Ma, Sci. Bull. 64, 310 (2019).

[25] D. M. Kennes, J. Lischner, and C. Karrasch, Phys. Rev. B 98, 241407 (2018).

[26] C.-C. Liu, L.-D. Zhang, W.-Q. Chen, and F. Yang, Phys. Rev. Lett. 121, 217001 (2018).
[27] L. Rademaker and P. Mellado, Phys. Rev. B 98, 235158 (2018),

[28] J. W. F. Venderbos and R. M. Fernandes, Phys. Rev. B 98 , 245103 (2018).

[29] H. Guo, X. Zhu, S. Feng, and R. T. Scalettar, Phys. Rev. B 97, 235453 (2018).

[30] Y.-P. Lin and R. M. Nandkishore, Phys. Rev. B 98, 214521 (2018).

[31] L. Zhang, Sci. Bull. 64, 495 (2019).

[32] M. Fidrysiak, M. Zegrodnik, and J. Spałek, Phys. Rev. B 98, 085436 (2018).

[33] B. Roy and V. Juricic, Phys. Rev. B 99, 121407 (2019).

[34] Y. Su and S.-Z. Lin, Phys. Rev. B 98, 195101 (2018).

[35] S. Ray and T. Das, Phys. Rev. B 99, 134515 (2019).

[36] L. Zou, H. C. Po, A. Vishwanath, and T. Senthil, Phys. Rev. B 98, 085435 (2018).

[37] H. C. Po, L. Zou, T. Senthil, and A. Vishwanath, Phys. Rev. B 99, 195455 (2019).

[38] Q. Tang, L. Yang, D. Wang, F. Zhang, and Q. Wang, Phys. Rev. B 99, 094521 (2019).

[39] Y.-Z. You and A. Vishwanath, arXiv:1805.06867.

[40] G. Baskaran, arXiv:1804.00627.

[41] T. J. Peltonen, R. Ojajärvi, and T. T. Heikkilä, Phys. Rev. B 98 , 220504 (2018).

[42] V. Y. Irkhin and Y. N. Skryabin, JETP Lett. 107, 651 (2018).

[43] F. Wu, A. MacDonald, and I. Martin, Phys. Rev. Lett. 121, 257001 (2018)

[44] S. Carr, S. Fang, P. Jarillo-Herrero, and E. Kaxiras, Phys. Rev. B 98, 085144 (2018).

[45] F. Guinea and N. R. Walet, Proc. Natl. Acad. Sci. USA 115 , 13174 (2018)

[46] B. Lian, F. Xie, and B. A. Bernevig, arXiv:1811.11786.

[47] B. Lian, Z. Wang, and B. A. Bernevig, Phys. Rev. Lett. 122, 257002 (2019).

[48] Z. Song, Z. Wang, W. Shi, G. Li, C. Fang, and B. A. Bernevig, Phys. Rev. Lett. 123, 036401 (2019).

[49] J. Ahn, S. Park, and B.-J. Yang, Phys. Rev. X 9, 021013 (2019).

[50] E. Laksono, J. N. Leaw, A. Reaves, M. Singh, X. Wang, S. Adam, and X. Gu, Solid State Commun. 282, 38 (2018).

[51] L. Chen, H.-Z. Li, and R.-S. Han, J. Phys.: Condens. Matter 31, 065601 (2018).

[52] Z. Liu, Y. Li, and Y.-f. Yang, Chin. Phys. B 28, 077103 (2019).

[53] A. Kerelsky, L. McGilly, D. M. Kennes, L. Xian, M. Yankowitz, S. Chen, K. Watanabe, T. Taniguchi, J. Hone, C. Dean et al., Nature (London) 572, 95 (2019).

[54] Y. Choi, J. Kemmer, Y. Peng, A. Thomson, H. Arora, R. Polski, Y. Zhang, H. Ren, J. Alicea, G. Refael et al., Nat. Phys. 15, 1174 (2019).

[55] J. Liu, J. Liu, and X. Dai, Phys. Rev. B 99, 155415 (2019).

[56] R. Bistritzer and A. H. MacDonald, Proc. Natl. Acad. Sci. USA 108, 12233 (2011).

[57] B. Hunt, J. D. Sanchez-Yamagishi, A. F. Young, M. Yankowitz, B. J. LeRoy, K. Watanabe, T. Taniguchi, P. Moon, M. Koshino, P. Jarillo-Herrero, and R. C. Ashoori, Science 340, 1427 (2013). 
[58] D. Xiao, M.-C. Chang, and Q. Niu, Rev. Mod. Phys. 82, 1959 (2010).

[59] Y.-H. Zhang and T. Senthil, Phys. Rev. B 99, 205150 (2019).

[60] J. Kang and O. Vafek, Phys. Rev. Lett. 122, 246401 (2019).

[61] Because of Coulomb interaction and the size of each peak for the fidget spinner, the detailed parameters for the interaction may deviate from this simple expression.

[62] Note that as there is spin SU(2) symmetry, spontaneous spin polarization will not lead to hysteresis; the valley polarization, however, is an Ising order parameter and hence hysteresis is expected.
[63] L. B. Ioffe and A. I. Larkin, Phys. Rev. B 39, 8988 (1989).

[64] Y.-H. Zhang, H. C. Po, and T. Senthil, Phys. Rev. B 100, 125104 (2019).

[65] X. Lu, P. Stepanov, W. Yang, M. Xie, M. A. Aamir, I. Das, C. Urgell, K. Watanabe, T. Taniguchi, G. Zhang et al., Nature (London) 574, 653 (2019).

[66] N. Bultinck, S. Chatterjee, and M. P. Zaletel, arXiv:1901.08110.

[67] N. Marzari, A. A. Mostofi, J. R. Yates, I. Souza, and D. Vanderbilt, Rev. Mod. Phys. 84, 1419 (2012).

[68] B. I. Halperin, P. A. Lee, and N. Read, Phys. Rev. B 47, 7312 (1993). 